\title{
Laser Scanning Confocal Microscopy 3D Surface Metrology Applications
}

\author{
Mina Hong ${ }^{1}$ and Guangnan Meng $^{1}$ \\ 1. Olympus America Inc. Scientific Solutions, Waltham, MA, USA
}

Surface metrology, measurement of solid surfaces topography, has become an important topic for many material scientists and engineers. Characterization of the surfaces can help researchers find new functional materials, improve device performance and so forth. 'Seeing is believing.' Visualization of fine 3-dimensional (3D) details of surfaces is critical in surface metrology studies. Among various observation and measurement techniques, Laser Scanning Confocal Microscopy (LSCM) becomes more and more popular due to the fact that it is non-contact and non-destructive to the samples, requires minimal sample preparation, and efficient automations and provides single nanometer level resolution. In this paper, we will introduce these features in detail, by using Olympus LEXT OLS5000, the newest 3D laser confocal scanning microscope.

LCSM utilizes a single short wavelength laser beam (normally 405nm) as the light source. By employing pinhole apertures to remove out-of-focus haze, it can dramatically increase contrast and resolution. Combing in-plane point by point scan and $\mathrm{z}$ axis stack scan, LCSM can assemble all $\mathrm{x}$, $\mathrm{y}$ and $\mathrm{z}$ for each pixel and turn into a 3D mapping. The lateral resolution of LEXT OLS5000 is $\sim 200 \mathrm{~nm}$ and vertical resolution can be $6 \mathrm{~nm}$ only.

Using laser as the feedback signal, without any physical contact of samples, LSCM is a truly noncontact and nondestructive technique. No sample preparation is required and all samples can be well preserved from any abrasion or contamination. LSCM can be widely applied from small chips to large tools, silicon to soft sponges.

With the open design of the system, LEXT OLS5000 can accommodate large samples easily. It can hold samples as high as $210 \mathrm{~mm}$, and the ultra-long working distance objectives facilitates measurements of concavities as deep as $25 \mathrm{~mm}$. In addition, the scan size equaling the field of view is only determined by the magnification of the objective lenses. $100 \mathrm{X}$ objective lenses provides a $128 \mu \mathrm{m} \times 128 \mu \mathrm{m}$ field of view and $5 \mathrm{X}$ with $2570 \mu \mathrm{m} \times 2570 \mu \mathrm{m}$. It only takes a few seconds to finish one image. With the motorized stage, it is easy and straightforward to stich high resolution images. LEXT OLS5000 can stich up to a size of $10 \mathrm{~cm} \times 10 \mathrm{~cm}$ image.

LCSM can precisely measures shape, sidewall and surface roughness at the submicron level. Surface roughness indicating the flatness of the sample is a very important parameter in metrology. LSCM can provide line roughness and area roughness data of the samples from 3D mapping. It uses laser spot with only a few hundred $\mathrm{nm}$ and enables operators to specify the measurement line or area of interest after data acquisition from the surface (Figure 1). Measurements of sidewall features (line, trench or contact holes), angles or roughness are important for certain wafers and trench fillings [1]. Cross section Scanning Electron Microscope (SEM), tilting stage combined AFM and critical dimension SEM are common tools to obtain sidewall information. All these tools are both time consuming and complicated to use compared to LSCM. LEXT OLS5000 can resolve sidewall with angels up to $87.5^{\circ}$ without addition cost of time and hardware. Surface roughness data can be provided the same time (Figure 2). 
In all, LSCM, with the capability to make accurate 3D measurements on a wide range of sample types and applications, delivers reliable data for quality assurance and process control. It provides 3D structure, sidewall measurement, roughness data and so forth as easy as a digital microscope taking snapshots of the samples thus is a nondestructive, precise, fast and easy way for your 3D metrology applications.

\section{References:}

[1] B. Su et al, IEEE Xplore (2002).

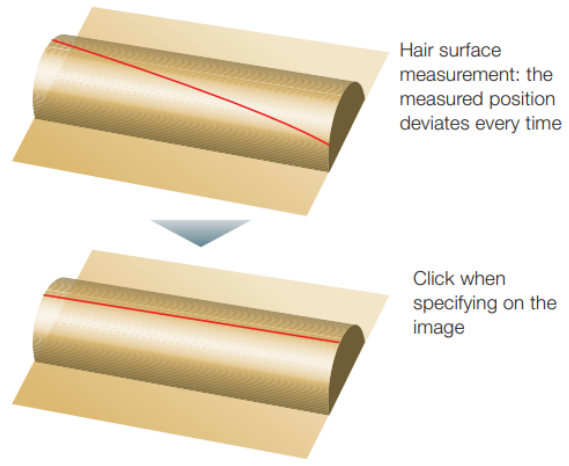

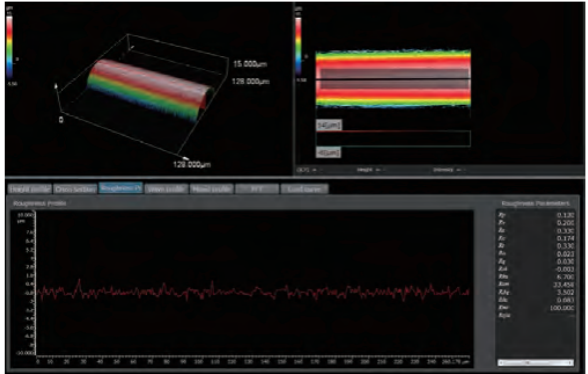

(a)

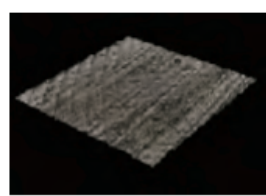

Color image

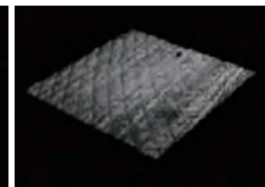

Laser image

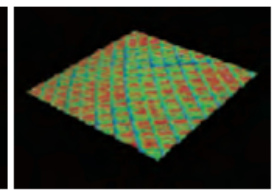

3D shape data

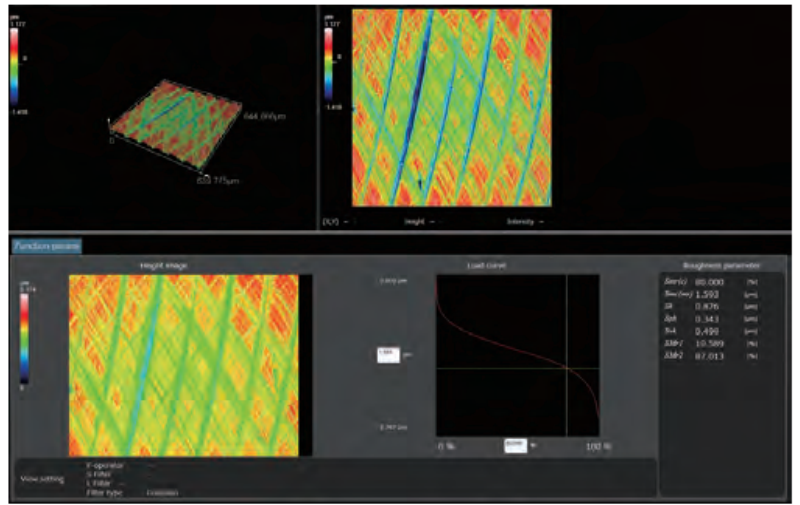

(b)

Figure 1. Olympus OLS5000 LCSM easily reveals the (a) line roughness on a tube sample and (b) surface roughness of a skin sample.

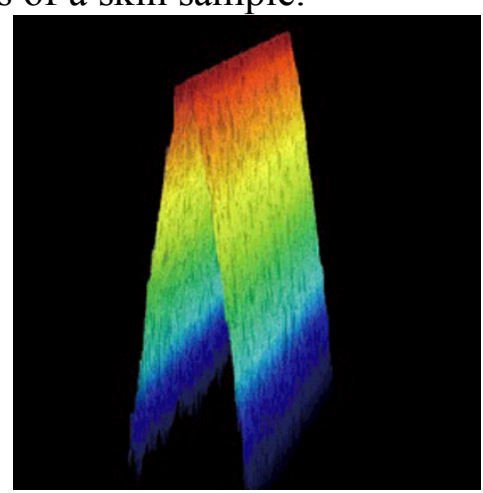

(a)

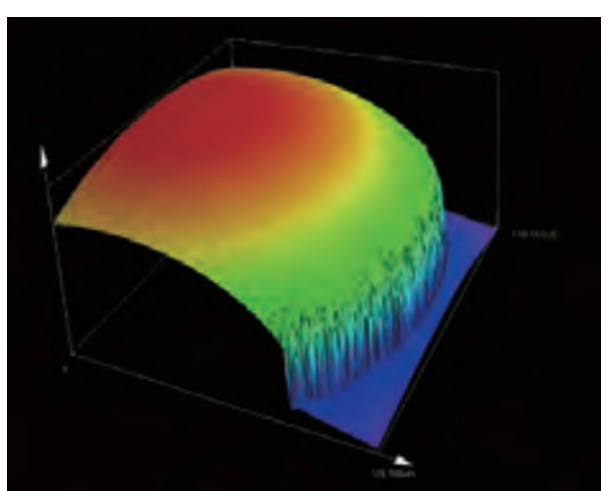

(b)

Figure 2. Olympus OLS5000 LCSM accurately detects slopes as steep as $87.5^{\circ}$. (a) Blade edge and (b) ruby ball. 